\title{
Proton-Induced Lewis Acidity of Unsaturated Iridium Amides
}

Zachariah M. Heiden and Thomas B. Rauchfuss*

School of Chemical Sciences

University of Illinois

Urbana, IL 61801

RECEIVED DATE (automatically inserted by publisher); rauchfuz@uiuc.edu

\section{Supporting Information}

Index

1) Experimental procedures

2) $\mathrm{pK}_{\mathrm{a}}$ determination of $[1 \mathrm{H}] \mathrm{BAr}_{4}^{\mathrm{F}} \quad \mathrm{S} 4$

3) Single crystal X-ray analysis for $[1 \mathrm{H}] \mathrm{BAr}^{\mathrm{F}}{ }_{4} \cdot \mathrm{H}_{2} \mathrm{O} \cdot 2.5 \mathrm{Et}_{2} \mathrm{O} \quad \mathrm{S} 5$

$\begin{array}{ll}\text { 4) Single crystal X-ray analysis for } 2 & \text { S7 }\end{array}$

$\begin{array}{ll}\text { 5) } & \text { References }\end{array}$

\section{1) Preparative methods and properties}

Electrochemical Measurements of IrHCp*(TsDPEN). Cyclic voltammograms were measured using a BAS CV-50W electrochemical analyzer at a scan rate of 250 $\mathrm{mV} / \mathrm{s}$ over a potential range of $4200 \mathrm{mV}$, starting at $-2600 \mathrm{mV}$, on $1.0 \times 10^{-3} \mathrm{M}$ acetonitrile solutions of $\mathrm{Cp}^{*} \mathrm{IrH}(\mathrm{TsDPEN})^{1}$ using $0.10 \mathrm{M} \mathrm{Bu}_{4} \mathrm{NPF}_{6}$ as supporting electrolyte and referenced to an external $\mathrm{Fc} / \mathrm{Fc}^{+}$reference $\left(\mathrm{E}^{\circ}=0.43 \mathrm{~V}\right.$ vs. $\left.\mathrm{Ag} / \mathrm{Ag}^{+}\right)$. A platinum wire counter electrode, a glassy carbon working electrode and a $\mathrm{Ag} / \mathrm{AgCl}(\mathrm{aq})$ reference electrode were used.

[Cp*Ir(TsDPEN)(NCMe)]PF 6 ([1H(NCMe)]PF 6 ). A solution of $0.10 \mathrm{~g}(0.14$ mmol) $\mathrm{Cp}^{*} \mathrm{IrH}(\mathrm{TsDPEN})^{1}$ in $10 \mathrm{~mL}$ of $\mathrm{MeCN}$ was treated with a solution of $45 \mathrm{mg}(0.14$ mmol) of $\mathrm{Cp}_{2} \mathrm{FePF}_{6}$ in $10 \mathrm{~mL} \mathrm{MeCN}$. Gas evolution was observed in most cases, accompanying a color change from orange to yellow. The resulting solution was evaporated to dryness and the solid residue was washed with hexane followed by $\mathrm{Et}_{2} \mathrm{O}$ to remove $\mathrm{Cp}_{2} \mathrm{Fe}$, which was identified by ${ }^{1} \mathrm{H} \mathrm{NMR}$ spectroscopy. Under a vacuum, solid samples of $[1 \mathrm{H}(\mathrm{NCMe})] \mathrm{PF}_{6}$ become bright red, indicating the conversion to $[1 \mathrm{H}] \mathrm{PF}_{6}$. The identification of the cyclometalated species was verified by a separate preparation of 2. $[1 \mathrm{H}(\mathrm{NCMe})] \mathrm{PF}_{6}$ was recrystallized by dissolution in $\mathrm{MeCN}$ followed by the addition 
of $\mathrm{Et}_{2} \mathrm{O}$. Yield: $65 \mathrm{mg}(53 \%)$. ${ }^{1} \mathrm{H}$ NMR $\left(500 \mathrm{MHz}, \mathrm{CD}_{3} \mathrm{CN}\right): \delta 1.82$ (s, 15H, Cp*), 1.96 (s, $3 \mathrm{H}, \mathrm{CH}_{3} \mathrm{CN}$ ), 2.18 (s, 3H, $\mathrm{SO}_{2} \mathrm{C}_{6} \mathrm{H}_{4}-4-\mathrm{CH}_{3}$ ), 3.68 (br t, $1 \mathrm{H}, 11 \mathrm{~Hz}$, $\mathrm{H}_{2} \mathrm{NCHPhCHPhNTs}$ ), 4.38 (m, 1H, $11 \mathrm{~Hz}, \mathrm{H} H \mathrm{NCHPhCHPhNTs}$ ), 4.42 (d, 1H, $11 \mathrm{~Hz}$, $\mathrm{H}_{2} \mathrm{NCHPhCHPhNTs}$ ), 5.18 (br d, 1H, 9.4 Hz, HHNCHPhCHPhNTs), 6.62-7.26 (m, $14 \mathrm{H})$.

Measurement of Hydrogen Production from Oxidation of $\mathbf{1 H}(\mathrm{H})$. The pressure difference resulting from the oxidation of $1 \mathrm{H}(\mathrm{H})$ in $\mathrm{MeOH}$ solution was measured using a modified Toepler pump. The moles of hydrogen produced were backcalculated using the Ideal Gas and van der Waals equations of state; the same value to three significant figures resulted from both equations. The solubility of $\mathrm{H}_{2}$ in methanol using Henry's Law was found to be negligible to three significant figures. ${ }^{2}$ An $87.3 \%$ recovery of the theoretical $\mathrm{H}_{2}$ was observed, confirming the stoichiometry of $1 / 2 \mathrm{~mol} \mathrm{H}_{2}$ per mol of 1 .

Oxidation of $\mathbf{1 H}(\mathbf{H})$ with $\mathbf{P h}_{3} \mathbf{C P F}_{6}$. A solution of $50 \mathrm{mg}(0.07 \mathrm{mmol})$ $\mathrm{Cp}^{*} \operatorname{IrH}(\mathrm{TsDPEN})$ in $10 \mathrm{~mL}$ of MeCN was treated with a solution of $25 \mathrm{mg}(0.06 \mathrm{mmol})$ of $\mathrm{Ph}_{3} \mathrm{CPF}_{6}$ in $10 \mathrm{~mL} \mathrm{MeCN}$. A color change from orange to yellow was observed. The solvent was removed under reduced pressure. The resulting solid was identified by ${ }^{1} \mathrm{H}$ NMR spectroscopy verifying the presence of $[\mathbf{1 H}(\mathrm{NCMe})] \mathrm{PF}_{6}$ and $\mathbf{2}$. The formation of 2 was found to account for $13 \%$ of the Ir products, the remainder being [1 $\mathrm{H}(\mathrm{NCMe})] \mathrm{PF}_{6}$. The presence of $\mathrm{Ph}_{3} \mathrm{CH}$ was verified by a singlet at $\delta 5.61\left(500 \mathrm{MHz}, \mathrm{CD}_{3} \mathrm{CN}\right)$; the presence of Gomberg's dimer was not observed. ${ }^{3}$

Alternative synthesis of 2 from $C p^{*} \operatorname{Ir}(T s D P E N-H)(1)$. A solution of $0.1 \mathrm{~g}(0.14$ mmol) $\mathrm{Cp} * \operatorname{Ir}(\mathrm{TsDPEN}-\mathrm{H})^{1}$ in $15 \mathrm{~mL} \mathrm{MeOH}$ was heated at reflux for $4 \mathrm{~h}$. The solvent was removed under reduced pressure resulting in a reddish yellow solid. The solid was recrystallized by dissolution in $\mathrm{MeOH}$ followed by the addition of $\mathrm{Et}_{2} \mathrm{O}$, giving yellow crystals. Yield: $63 \mathrm{mg}(63 \%)$. The yield decreased upon stirring for extended time periods before reflux. ${ }^{1} \mathrm{H}$ NMR $\left(500 \mathrm{MHz}, \mathrm{CD}_{3} \mathrm{CN}\right): \delta 1.88(\mathrm{~s}, 15 \mathrm{H}, 1 \mathrm{~Hz}, \mathrm{Cp} *), 2.21$ (s, $3 \mathrm{H}, \mathrm{SO}_{2} \mathrm{C}_{6} \mathrm{H}_{4}-4-\mathrm{CH}_{3}$ ), 3.36 (br d, $1 \mathrm{H}, 7 \mathrm{~Hz}, \mathrm{HHNCH}\left(\mathrm{C}_{6} \mathrm{H}_{4}\right) \mathrm{CHPhNTs}$ ), 3.51 (br d, 1H, 8 $\left.\mathrm{Hz}, \mathrm{H} H \mathrm{NCH}\left(\mathrm{C}_{6} \mathrm{H}_{4}\right) \mathrm{CHPhNTs}\right), 3.88$ (s, $\left.1 \mathrm{H}, \mathrm{H}_{2} \mathrm{NCH}\left(\mathrm{C}_{6} \mathrm{H}_{4}\right) \mathrm{CHPhNTs}\right), 4.39$ (s, 1H, $\left.\mathrm{H}_{2} \mathrm{NCH}\left(\mathrm{C}_{6} \mathrm{H}_{4}\right) \mathrm{CHPhNTs}\right), 6.63$ (m, 2H, $\left.\mathrm{C}_{6} \mathrm{H}_{4}\right), 6.75$ (d, 2H, $\left.8 \mathrm{~Hz}, \mathrm{Ts}\right), 6.90$ (d, 2H, $8 \mathrm{~Hz}$, Ts) $6.99\left(\mathrm{dd}, 1 \mathrm{H}, 2,7 \mathrm{~Hz}, \mathrm{C}_{6} H_{4}\right), 7.29\left(\mathrm{t}, 1 \mathrm{H}, 7 \mathrm{~Hz}, \mathrm{C}_{6} \mathrm{H}_{4}\right), 7.41$ (q, 3H, $\left.8 \mathrm{~Hz}, \mathrm{Ph}\right), 7.61$ (d, 2H, $7 \mathrm{~Hz}, \mathrm{Ph}$ ). Anal. Calcd for $\mathrm{C}_{31} \mathrm{H}_{35} \mathrm{IrN}_{2} \mathrm{O}_{2} \mathrm{~S}: \mathrm{C}, 53.81 ; \mathrm{H}, 5.06 ; \mathrm{N}, 4.05$. Found: $\mathrm{C}$, $54.19 ; \mathrm{H}, 4.85 ; \mathrm{N}, 3.80$.

$[\mathrm{Cp} * \operatorname{Ir}(\mathrm{TsDPEN})(\mathrm{NCMe})] \mathrm{BAr}^{\mathrm{F}}{ }_{4}\left([\mathbf{1 H}(\mathbf{N C M e})] \mathrm{BAr}^{\mathrm{F}}{ }_{4}\right)$. A solution of $0.21 \mathrm{~g}$ $(0.3 \mathrm{mmol}) \mathrm{Cp}^{*} \operatorname{Ir}(\mathrm{TsDPEN}-\mathrm{H})$ in $10 \mathrm{~mL}$ of MeCN was treated with $0.31 \mathrm{~g}(0.3 \mathrm{mmol})$ of $\mathrm{H}\left(\mathrm{OEt}_{2}\right)_{2} \mathrm{BAr}^{\mathrm{F}}{ }_{4}$. An immediate color change from purple to yellow was observed. After the solution stirred for $30 \mathrm{~min}$, the solvent was removed under reduced pressure. The resulting red solid was washed with $30 \mathrm{~mL}$ hexane. Yield: $0.39 \mathrm{~g}(83 \%)$. ${ }^{1} \mathrm{H}$ NMR (500 $\mathrm{MHz}, \mathrm{CD}_{3} \mathrm{CN}$ ): $\delta 1.82$ (s, 15H, Cp*), 2.18 (s, 3H, $\mathrm{SO}_{2} \mathrm{C}_{6} \mathrm{H}_{4}-4-\mathrm{CH}_{3}$ ), 3.68 (br t, $1 \mathrm{H}, 11$ $\mathrm{Hz}, \mathrm{H}_{2} \mathrm{NCHPhCHPhNTs}$ ), 4.38 (m, 1H, $11 \mathrm{~Hz}, \mathrm{H} H \mathrm{NCHPhCHPhNTs}$ ), 4.42 (d, 1H, 11 $\mathrm{Hz}, \mathrm{H}_{2} \mathrm{NCHPhCHPhNTs}$ ), 5.18 (br d, 1H, 9.4 Hz, HHNCHPhCHPhNTs), 6.62-7.26 (m, $14 \mathrm{H}), 7.66-7.71\left(\mathrm{~s}, 12 \mathrm{H}, \mathrm{BAr}^{\mathrm{F}}{ }_{4}\right)$.

Cp* $\operatorname{Ir}\left(\mathbf{T s N C H}(\mathbf{P h}) \mathbf{C H}(\mathbf{P h}) \mathbf{N D}_{2}\right) \mathbf{H}\left(\mathbf{1 H}(\mathbf{H})-\boldsymbol{d}_{2}\right)$. A volume of $3 \mathrm{~mL}$ of $\mathrm{CH}_{3} \mathrm{OD}$ ( $80.8 \mathrm{mmol})$ was added to $0.2 \mathrm{~g}(0.3 \mathrm{mmol}) \mathrm{Cp} * \operatorname{Ir}(\mathrm{TsDPEN}-\mathrm{H})$ with stirring. The resulting reddish-purple colored solution was allowed to stir for $10 \mathrm{~min}$. An orange solid was observed to form after $4 \mathrm{~min}$. The solution was allowed to cool for $2 \mathrm{~h}$ at $-40^{\circ} \mathrm{C}$. 
The orange solid was collected by filtration and washed twice with $\mathrm{Et}_{2} \mathrm{O}$ yielding orange microcrystals. Yield: $99 \mathrm{mg}(50 \%)$. ${ }^{1} \mathrm{H}$ NMR (500 MHz, $\left.\mathrm{CD}_{3} \mathrm{CN}\right): \delta-10.76$ (s, $\left.1 \mathrm{H}, \mathrm{Ir} H\right)$, 1.87 (d, 15H, $1 \mathrm{~Hz}, \mathrm{Cp}^{*}$ ), 2.37 (s, 3H, SO $\mathrm{C}_{6} \mathrm{H}_{4}-4-M e$ ), 3.68 (br d, 1H, $9.2 \mathrm{~Hz}$, $\mathrm{H}_{2} \mathrm{NCHPhCHPhNTs}$ ), 4.14 (d, 1H, 9.2 Hz, H $\mathrm{H}_{2} \mathrm{NCHPhCHPhNTs}$ ), 6.86-7.20 (12H), 7.60 (d, $2 \mathrm{H}, 8 \mathrm{~Hz}, \mathrm{H}_{2} \mathrm{NCHPhCHPhNTs}$ ). Oxidation of this material using $\mathrm{FcPF}_{6}$ in $\mathrm{CD}_{3} \mathrm{CN}$ resulted in exclusive formation of $\mathrm{H}_{2}$ and $\left[\mathbf{1 H}\left(\mathrm{NCCD}_{3}\right)-d_{2}\right] \mathrm{PF}_{6}$.

Deuteration of Cp* IrH(TsDPEN) in $\mathbf{C D}_{3}$ OD. A solution containing $20 \mathrm{mg}$ $(0.03 \mathrm{mmol}) \mathrm{Cp} * \mathrm{IrH}(\mathrm{TsDPEN})$ and $5 \mathrm{mg}(0.06 \mathrm{mmol}) \mathrm{C}_{6} \mathrm{Me}_{6}$ (internal standard) in 0.75 mL $\mathrm{CD}_{3} \mathrm{OD}$ was monitored via ${ }^{1} \mathrm{H}$ NMR spectroscopy. The rate of deuteration was calculated from the rate of decrease in the integration values of the amine and hydride peaks with respect to the internal standard. Spectra were obtained in five-minute intervals for duration of 70 minutes from the addition of $\mathrm{CD}_{3} \mathrm{OD}$. The amine protons were estimated to have an exchange rate of $0.00015 \mathrm{~s}^{-1}$, and the hydride was estimated to have an exchange rate of $0.00003 \mathrm{~s}^{-1}$.

$\left[\mathbf{C p}^{*} \mathbf{I r}(\mathbf{T s D P E N})\right] \mathbf{B A r}^{\mathrm{F}}{ }_{4}\left([1 \mathbf{H}] \mathbf{B A r}^{\mathbf{F}}{ }_{4}\right)$. A solution of $0.30 \mathrm{~g}(0.3 \mathrm{mmol})$ $\mathrm{H}\left(\mathrm{OEt}_{2}\right)_{2} \mathrm{BAr}_{4}{ }_{4}$ in $10 \mathrm{~mL} \mathrm{CH}_{2} \mathrm{Cl}_{2}$ was added to a solution of $0.20 \mathrm{~g}(0.3 \mathrm{mmol})$ $\mathrm{Cp}$ * $\operatorname{Ir}(\mathrm{TsDPEN}-\mathrm{H})$ in $\mathrm{CH}_{2} \mathrm{Cl}_{2}$. The resulting red solution was allowed to stir for $30 \mathrm{~min}$. The solvent was removed under reduced pressure. The resulting red-colored solid was washed twice with $20 \mathrm{~mL}$ n-hexane. Yield: $0.28 \mathrm{~g}(60 \%) .{ }^{1} \mathrm{H} \mathrm{NMR}\left(500 \mathrm{MHz}, \mathrm{CD}_{3} \mathrm{OD}\right)$ : $\delta 1.95$ (s, 15H, Cp*), 2.30 (s, 3H, $\mathrm{SO}_{2} \mathrm{C}_{6} \mathrm{H}_{4}-4-\mathrm{CH}_{3}$ ), 4.25 (s, 1H, $\mathrm{H}_{2} \mathrm{NCHPhCHPhNTs}$ ), 4.64 (s, 1H, $\left.\mathrm{H}_{2} \mathrm{NCHPhCHPhNTs}\right), 6.92-7.43$ (m, 14H), 7.59 (s, 12H, BArF 4$) .{ }^{1} \mathrm{H} \mathrm{NMR}$ $\left(500 \mathrm{MHz}, \mathrm{CD}_{2} \mathrm{Cl}_{2}\right): \delta 1.83$ (s, 15H, Cp*), 2.28 (s, 3H, $\left.\mathrm{SO}_{2} \mathrm{C}_{6} \mathrm{H}_{4}-4-\mathrm{CH}_{3}\right), 4.18$ (br d, $1 \mathrm{H}$, $10 \mathrm{~Hz}, \mathrm{H} H \mathrm{NCHPhCHPhNTs}$ ), 4.31 (s, $\mathrm{H}_{2} \mathrm{NCHPhCHPhNTs}$ ), 4.40 (br d, 1H, $10 \mathrm{~Hz}$, HHNCHPhCHPhNTs), 4.91 (s, 1H, $\mathrm{H}_{2} \mathrm{NCHPhCHPhNTs),} \mathrm{6.91-7.35} \mathrm{(14H),} \mathrm{7.57-7.73}$ $\left(12 \mathrm{H}, \mathrm{BArF}_{4}\right)$. Anal. Calcd for $\mathrm{C}_{63} \mathrm{H}_{48} \mathrm{BF}_{24} \mathrm{IrN}_{2} \mathrm{O}_{2} \mathrm{~S}: \mathrm{C}, 48.63 ; \mathrm{H}, 3.11 ; \mathrm{N}, 1.80$. Found: C, 48.70; H, 3.15; N, 2.09. ESI-MS: $\mathrm{m} / \mathrm{z}=693\left(\mathrm{MH}^{+}\right)$.

The $\mathrm{NH}_{3}, \mathrm{PPh}_{3}$, and $\mathrm{CO}$ adducts were first prepared employing $\mathrm{BAr}_{4}^{F_{4}}$ as a counteranion, but difficulty in isolation led to the use of $\mathrm{BF}_{4}$ in scaled-up preparations.

$\left[\mathbf{C p} * \operatorname{Ir}\left(\mathbf{T s D P E N}_{\mathbf{2}}\left(\mathbf{N H}_{3}\right)\right] \mathbf{B A r}^{\mathbf{F}}{ }_{\mathbf{4}}\left(\left[\mathbf{1} \mathbf{H}\left(\mathbf{N H}_{3}\right)\right] \mathbf{B A r}^{\mathbf{F}}{ }_{4}\right)\right.$. A solution of $10 \mathrm{mg}(0.01$ mmol) $[\mathrm{Cp} * \operatorname{Ir}(\mathrm{TsDPEN})] \mathrm{BAr}^{\mathrm{F}}{ }_{4}$ and $5 \mathrm{mg}(0.06 \mathrm{mmol}) \mathrm{C}_{6} \mathrm{Me}_{6}$ (internal standard) in 0.75 $\mathrm{mL} \mathrm{CD}_{3} \mathrm{CN}$ was shaken with $1.0 \mathrm{~mL}$ of 1 atm of $\mathrm{NH}_{3}$. A color change from yellow to a pale yellow was observed. Yield: Quantitative. ${ }^{1} \mathrm{H} \mathrm{NMR}\left(500 \mathrm{MHz}, \mathrm{CD}_{3} \mathrm{CN}\right): \delta 1.77$ (s, 15H, Cp*), 2.15 (s, 3H, SO $\mathrm{C}_{6} \mathrm{H}_{4}-4-\mathrm{CH}_{3}$ ), 3.76 (br t, $1 \mathrm{H}, 11 \mathrm{~Hz}, \mathrm{H}_{2} \mathrm{NCHPhCHPhNTs}$ ), 3.90 (br s, 3H, NH $H_{3}$ ), 4.30 (br t, 1H, 13 Hz, HHNCHPhCHPhNTs), 4.54 (d, 1H, 10.7 Hz, $\mathrm{H}_{2} \mathrm{NCHPhCHPhNTs}$ ), 5.24 (br d, 1H, $11 \mathrm{~Hz}, H \mathrm{HNCHPhCHPhNTs}$ ), 6.60-6.81 (7H), 7.10-7.23 (7H), 7.65-7.73 (12H, $\left.\mathrm{BAr}_{4}{ }_{4}^{-}\right)$.

$\left[\mathbf{C p} * \operatorname{Ir}(\mathbf{T s D P E N})\left(\mathbf{N H}_{3}\right)\right] \mathbf{B F}_{\mathbf{4}}\left(\left[\mathbf{1 H}\left(\mathbf{N H}_{3}\right)\right] \mathbf{B F}_{4}\right)$. A solution of $57 \mathrm{mg}(0.35 \mathrm{mmol})$ $\mathrm{NH}_{4} \mathrm{BF}_{4}$ in $10 \mathrm{~mL} \mathrm{MeCN}$ was added to a solution of $0.2 \mathrm{~g}(0.3 \mathrm{mmol}) \mathrm{Cp} * \operatorname{Ir}(\mathrm{TsDPEN}-\mathrm{H})$ in $\mathrm{MeCN}$. The resulting bright yellow solution was allowed to stir for $30 \mathrm{~min}$. The solvent was removed under reduced pressure resulting in a light yellow colored solid. The solid was washed with $20 \mathrm{~mL} \mathrm{Et}_{2} \mathrm{O}$ and recrystallized from $\mathrm{CH}_{2} \mathrm{Cl}_{2}$ solution by the addition of $\mathrm{Et}_{2} \mathrm{O}$. Yield: $0.18 \mathrm{~g}(69 \%)$. ${ }^{1} \mathrm{H} \mathrm{NMR}\left(500 \mathrm{MHz}, \mathrm{CD}_{3} \mathrm{CN}\right): \delta 1.77(\mathrm{~s}, 15 \mathrm{H}$, $\mathrm{Cp}^{*}$ ), 2.15 (s, 3H, $\mathrm{SO}_{2} \mathrm{C}_{6} \mathrm{H}_{4}-4-\mathrm{CH}_{3}$ ), 3.76 (br t, $1 \mathrm{H}, 11 \mathrm{~Hz}, \mathrm{H}_{2} \mathrm{NCHPhCHPhNTs}$ ), 3.85 (br s, 3H, NH $H_{3}$ ), 4.14 (t, 1H, 13 Hz, HHNCHPhCHPhNTs), 4.53 (d, 1H, $10.7 \mathrm{~Hz}$, $\mathrm{H}_{2} \mathrm{NCHPhCHPhNTs}$ ), 5.24 (br d, 1H, $11 \mathrm{~Hz}, H \mathrm{HNCHPhCHPhNTs}$ ), 6.60-6.81 (7H), 
7.10-7.22 (7H). Anal. Calcd for $\mathrm{C}_{31} \mathrm{H}_{39} \mathrm{BF}_{4} \mathrm{IrN}_{3} \mathrm{O}_{2} \mathrm{~S}$ : C, 46.73; H, 4.93; N, 5.27. Found: $\mathrm{C}, 43.51 ; \mathrm{H}, 4.45 ; \mathrm{N}, 4.32$. For $\left[1 \mathrm{H}\left(\mathrm{NH}_{3}\right)\right] \mathrm{BF}_{4} \mathrm{CH}_{2} \mathrm{Cl}_{2}: \mathrm{C}, 43.59 ; \mathrm{H}, 4.69 ; \mathrm{N}, 4.77$.

$\left[\mathbf{C p}\right.$ *Ir(TsDPEN)$\left.\left(\mathbf{P P h}_{3}\right)\right] \mathbf{B A r}^{\mathbf{F}}{ }_{4}\left(\left[\mathbf{1 H}\left(\mathbf{P P h}_{3}\right)\right] \mathbf{B A r}^{\mathbf{F}}{ }_{4}\right)$. A solution of $10 \mathrm{mg}(0.01$ mmol) $[\mathrm{Cp} * \operatorname{Ir}(\mathrm{TsDPEN})] \mathrm{BAr}{ }_{4}{ }_{4}$ and $5 \mathrm{mg}(0.06 \mathrm{mmol}) \mathrm{C}_{6} \mathrm{Me}_{6}$ (internal standard) in 0.75 $\mathrm{mL} \mathrm{CD} \mathrm{CN}_{3}$ was shaken with $5 \mathrm{mg}(0.02 \mathrm{mmol}) \mathrm{PPh}_{3}$. A color change from yellow to a bright yellow was observed. Yield: Quantitative. ${ }^{1} \mathrm{H}$ NMR $\left(500 \mathrm{MHz}, \mathrm{CD}_{3} \mathrm{CN}\right): \delta 1.57$ (d, 15H, $3 \mathrm{~Hz}, \mathrm{Cp}^{*}$ ), 2.17 (s, 3H, $\mathrm{SO}_{2} \mathrm{C}_{6} \mathrm{H}_{4}-4-\mathrm{CH}_{3}$ ), 3.59 (br t, $1 \mathrm{H}, 10 \mathrm{~Hz}$, $\mathrm{H}_{2} \mathrm{NCHPhCHPhNTs}$ ), 3.98 (br t, 1H, $13 \mathrm{~Hz}, \mathrm{H} H \mathrm{NCHPhCHPhNTs}$ ), 4.03 (d, 1H, $11 \mathrm{~Hz}$, $\mathrm{H}_{2} \mathrm{NCHPhCHPhNTs}$ ), 5.79 (d, 1H, $\left.11 \mathrm{~Hz}, H \mathrm{HNCHPhCHPhNTs}\right), 6.09$ (d, 2H, $11 \mathrm{~Hz}$, $\mathrm{H}_{2} \mathrm{NCHPhCHPhNTs}$ ), 6.63-8.23 (39H). ${ }^{31} \mathrm{P} \mathrm{NMR}\left(203 \mathrm{MHz}, \mathrm{CD}_{3} \mathrm{CN}\right): \delta 10.04$ (s).

$\left[\mathbf{C p} * \operatorname{Ir}\left(\mathbf{T s D P E N}_{\mathbf{S}}\left(\mathbf{P P h}_{\mathbf{3}}\right)\right] \mathbf{B F}_{\mathbf{4}}\left(\left[\mathbf{1 H}\left(\mathbf{P P h}_{3}\right)\right] \mathbf{B F}_{4}\right)\right.$. A solution of $47 \mathrm{mg}(0.18$ mmol $) \mathrm{PPh}_{3}$ in $10 \mathrm{~mL} \mathrm{MeCN}$ was added to a solution of $0.145 \mathrm{~g}(0.18 \mathrm{mmol})$ $\left[1 \mathrm{H}\left(\mathrm{NH}_{3}\right)\right] \mathrm{BF}_{4}$ in $\mathrm{MeCN}$. The resulting bright yellow solution was allowed to stir for 30 min. The solvent was removed under reduced pressure resulting in a bright yellow colored solid. The solid was recrystallized from $\mathrm{CH}_{2} \mathrm{Cl}_{2}$ by the addition of $\mathrm{Et}_{2} \mathrm{O}$. Yield: $0.15 \mathrm{~g}(80 \%)$. ${ }^{1} \mathrm{H}$ NMR (500 MHz, $\mathrm{CD}_{3} \mathrm{CN}$ ): $\delta 1.57$ (d, 15H, $\left.3 \mathrm{~Hz}, \mathrm{Cp}^{*}\right), 2.18$ (s, 3H, $\mathrm{SO}_{2} \mathrm{C}_{6} \mathrm{H}_{4}-4-\mathrm{CH}_{3}$ ), 3.59 (br t, $1 \mathrm{H}, 10 \mathrm{~Hz}, \mathrm{H}_{2} \mathrm{NCHPhCHPhNTs}$ ), 3.98 (br t, $1 \mathrm{H}, 13 \mathrm{~Hz}$, HHNCHPhCHPhNTs), 4.03 (d, 1H, 11 Hz, H $\mathrm{H}_{2} \mathrm{NCHPhCHPhNTs),} 5.79$ (d, 1H, $11 \mathrm{~Hz}$, HHNCHPhCHPhNTs), 6.09 (d, 2H, $\left.11 \mathrm{~Hz}, \mathrm{H}_{2} \mathrm{NCHPhCHPhNTs}\right), 6.63-8.23(27 \mathrm{H}) .{ }^{31} \mathrm{P}$ NMR (203 MHz, CD $\left.{ }_{3} \mathrm{CN}\right): \delta 10.04(\mathrm{~s})$. ESI-MS: $\mathrm{m} / \mathrm{z}=955.5\left(\mathrm{M}^{+}\right)$.

$[$ Cp*Ir(TsDPEN)(CO) $] B^{*} \mathbf{A r}^{\mathbf{F}}{ }_{4}\left([\mathbf{H}(\mathbf{C O})] \mathbf{B A r}^{\mathbf{F}}{ }_{4}\right)$. A solution of $10 \mathrm{mg}(0.01$ mmol) $\operatorname{IrCp}^{*}(\mathrm{TsDPEN}) \mathrm{BAr}_{4}{ }_{4}$ and $5 \mathrm{mg}(0.06 \mathrm{mmol}) \mathrm{C}_{6} \mathrm{Me}_{6}$ (internal standard) in 0.75 $\mathrm{mL} \mathrm{CD} \mathrm{Cl}_{2}$ was shaken with $1.0 \mathrm{~mL}$ of 1 atm of $\mathrm{CO}$. A color change from a red to a faint yellow was observed. Yield: Quantitative. ${ }^{1} \mathrm{H} \mathrm{NMR}\left(500 \mathrm{MHz}, \mathrm{CD}_{2} \mathrm{Cl}_{2}\right): \delta 2.08(\mathrm{~s}$, 15H, Cp*), 2.15 (s, 3H, SO $\mathrm{C}_{6} \mathrm{H}_{4}-4-\mathrm{CH}_{3}$ ), 3.59 (br t, 1H, $11 \mathrm{~Hz}, \mathrm{H}_{2} \mathrm{NCHPhCHPhNTs}$ ),

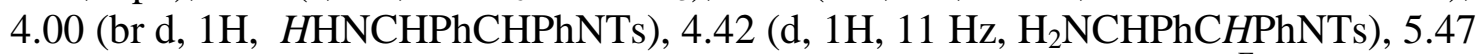
(br s, 1H, HHNCHPhCHPhNTs), 6.43-7.40 (14H), 7.55-7.75 (12H, $\left.\mathrm{BAr}_{4}{ }^{-}\right)$.

$\left[\mathbf{C p}^{*} \operatorname{Ir}\left(\mathrm{TsDPEN}_{\mathbf{2}}(\mathbf{C O})\right] \mathbf{B F}_{\mathbf{4}}\left([\mathbf{1 H}(\mathbf{C O})] \mathbf{B F}_{4}\right)\right.$. CO gas was bubbled through a solution of $0.20 \mathrm{~g}(0.25 \mathrm{mmol})[\mathrm{Cp} * \operatorname{Ir}(\mathrm{TsDPEN}-\mathrm{H})] \mathrm{BF}_{4}$ in $15 \mathrm{~mL} \mathrm{MeCN}$. The red colored solution became a faint yellow colored solution after 2 min., and the solution was allowed to stir for $30 \mathrm{~min}$. Solvent was removed under reduced pressure, and the product was recrystallized from $\mathrm{MeCN}$ solution by the addition of $\mathrm{Et}_{2} \mathrm{O}$. Yield: $0.14 \mathrm{~g} \mathrm{(75 \% ).}$ $v(\mathrm{CO})=2064 \mathrm{~cm}^{-1}\left(\mathrm{CH}_{2} \mathrm{Cl}_{2}\right) .{ }^{1} \mathrm{H} \mathrm{NMR}\left(500 \mathrm{MHz}, \mathrm{CD}_{3} \mathrm{CN}\right): \delta 2.04$ (s, 15H, Cp*), 2.17 (s, 3H, $\mathrm{SO}_{2} \mathrm{C}_{6} \mathrm{H}_{4}-4-\mathrm{CH}_{3}$ ), 3.51 (ddd, $1 \mathrm{H}, 3,11,13 \mathrm{~Hz}, \mathrm{H}_{2} \mathrm{NCHPhCHPhNTs}$ ), 4.37 (d, $1 \mathrm{H}, 11 \mathrm{~Hz}, \mathrm{H}_{2} \mathrm{NCHPhCHPhNTs}$ ), 5.07 (br d, 1H, $9 \mathrm{~Hz}, H \mathrm{HNCHPhCHPhNTs}$ ), 5.82 (br t, $1 \mathrm{H}, 9 \mathrm{~Hz}, \mathrm{H} H \mathrm{NCHPhCHPhNTs}), 6.57-7.27$ (14H). Anal. Calcd for $\mathrm{C}_{32} \mathrm{H}_{36} \mathrm{BF}_{4} \mathrm{IrN}_{2} \mathrm{O}_{3} \mathrm{~S}$ : C, 47.58; H, 4.49; N, 3.47. Found: C, 47.87, 48.62; H, 4.72, 4.58; N, 3.94, 3.09. ESIMS: $\mathrm{m} / \mathrm{z}=721.4\left(\mathrm{M}^{+}\right)$.

\section{2) $\mathrm{pK}_{\mathrm{a}}$ Determination of $[1 \mathrm{H}(\mathrm{NCMe})]^{+}$and $[1 \mathrm{H}]^{+}$.}

$\mathbf{p K}_{\mathbf{a}}$ of $[1 \mathbf{H}(\mathbf{N C M e})] \mathbf{B A r} \mathbf{F}_{4}{ }_{4}$ A $10 \mathrm{mg}(0.05 \mathrm{mmol})$ sample of proton sponge $\left(\mathrm{pK}_{\mathrm{a}}\right.$ $=18.62 \mathrm{MeCN})^{4}$ was added three times to a solution of $10 \mathrm{mg}(0.03 \mathrm{mmol})$ $\mathrm{Cp} * \operatorname{Ir}(\mathrm{NCMe})(\mathrm{TsDPEN}) \mathrm{BAr}{ }_{4}{ }_{4}$, and $5 \mathrm{mg}(0.06 \mathrm{mmol}) \mathrm{C}_{6} \mathrm{Me}_{6}$ (internal standard) in 0.75 $\mathrm{ml} \mathrm{CD}{ }_{3} \mathrm{CN}$, and was monitored via ${ }^{1} \mathrm{H}$ NMR spectroscopy. Only partial deprotonation was observed. The presence of $[\mathbf{1 H}(\mathrm{MeCN})]^{+}$and 1 were verified by ${ }^{1} \mathrm{H}$ NMR 
spectroscopy with their concentrations integrated using the $\mathrm{Cp} *$ peaks relative to $\mathrm{C}_{6} \mathrm{Me}_{6}$ $(\delta 2.18)$. A color change from yellow to light purple was observed upon subsequent additions of proton sponge. This experiment was repeated four times to determine the reproducibility of the $\mathrm{pK}_{\mathrm{a}}$ value, yielding $\mathrm{pK}_{\mathrm{a}}$ values of 21.23, 21.27, 21.14, and 21.13, respectively.

$\mathbf{p K}_{\mathrm{a}}$ of [1H] BAr $\mathbf{r}_{4}^{\mathbf{F}}$ in Methanol. Three, $1.0 \mu \mathrm{L}$, aliquots of $\mathrm{Et}_{3} \mathrm{~N}\left(\mathrm{pK}_{\mathrm{a}}=10.83\right.$ $\mathrm{MeOH})^{5}$ were added to a solution of $10 \mathrm{mg}(0.03 \mathrm{mmol})\left[\mathrm{Cp}^{*} \operatorname{Ir}(\mathrm{TsDPEN})\right] \mathrm{BAr}^{\mathrm{F}}{ }_{4}$, and 5 $\mathrm{mg}(0.06 \mathrm{mmol}) \mathrm{C}_{6} \mathrm{Me}_{6}$ (internal standard) in $0.75 \mathrm{~mL} \mathrm{CD}_{3} \mathrm{OD}$, and was monitored via ${ }^{1} \mathrm{H}$ NMR spectroscopy. The relative concentrations were determined by integration of the $\mathrm{Cp}^{*}$ peaks for $[\mathbf{1 H}]^{+}$and 1 relative to $\mathrm{C}_{6} \mathrm{Me}_{6}(\delta 2.19)$. This experiment was repeated twice to determine the reproducibility of the $\mathrm{pK}_{\mathrm{a}}$ value, and was verified by observation of complete deprotonation with piperidine $\left(\mathrm{pK}_{\mathrm{a}}=11.66\right.$ in $\left.\mathrm{MeOH}\right){ }^{5} \mathrm{~A} \mathrm{pK}_{\mathrm{a}}$ of $11.3 \pm 0.3$ was obtained for $[1 \mathrm{H}] \mathrm{BAr}_{4}^{\mathrm{F}}$ in $\mathrm{MeOH}$.

\section{3) Single crystal $\mathrm{x}$-ray diffraction of $[1 \mathrm{H}] \mathrm{BAr}^{\mathrm{F}}{ }_{4} \cdot \mathrm{H}_{2} \mathrm{O} \cdot 2.5 \mathrm{Et}_{2} \mathrm{O}$.}

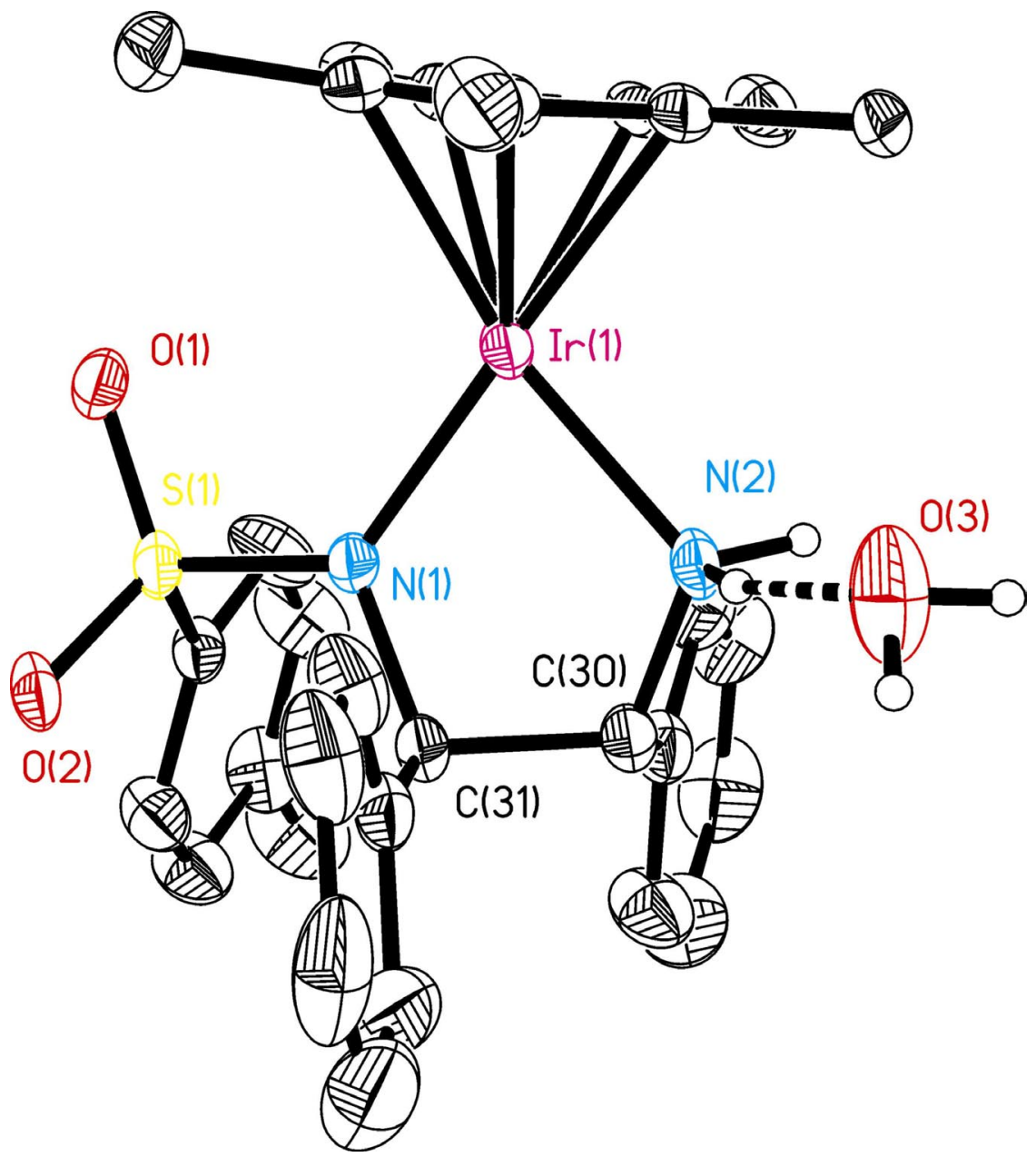

Figure S1: Molecular structure of $[1 \mathrm{H}] \mathrm{BAr}^{\mathrm{F}}{ }_{4} \cdot \mathrm{H}_{2} \mathrm{O} \cdot 2.5 \mathrm{Et}_{2} \mathrm{O}$ showing the hydrogen bonded water molecule. Disordered solvated ether molecules and $\mathrm{BArF}_{4}{ }^{-}$anion are omitted for clarity. Thermal ellipsoids are shown at $50 \%$ probability. 


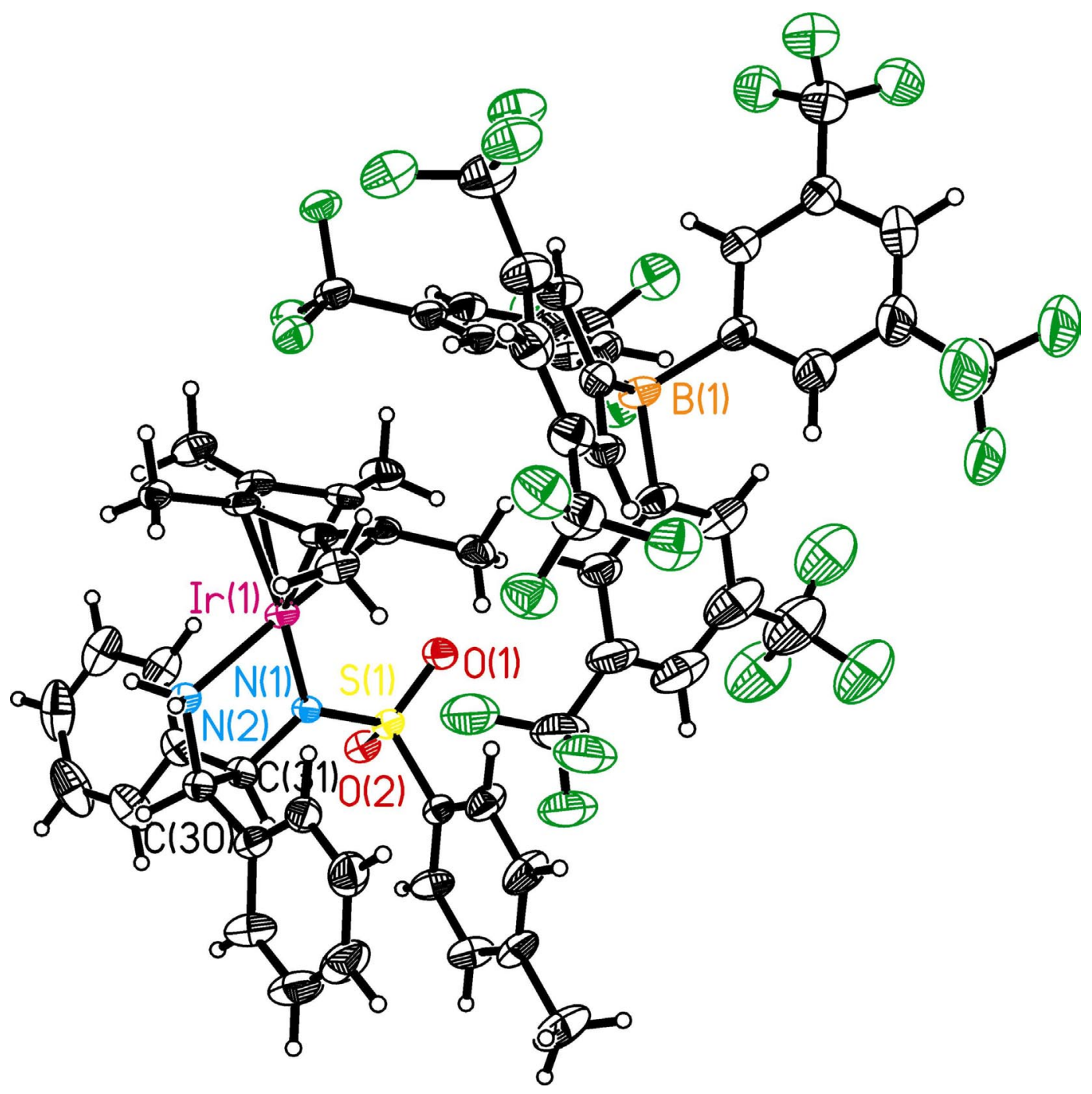

Figure S2: Molecular structure of $[1 \mathrm{H}] \mathrm{BAr}_{4}{ }_{4} \cdot \mathrm{H}_{2} \mathrm{O} \cdot 2.5 \mathrm{Et}_{2} \mathrm{O}$; solvated ether and water molecules are omitted for clarity. Thermal ellipsoids are shown at 50\% probability. 
4) Single crystal $x$-ray diffraction of 2

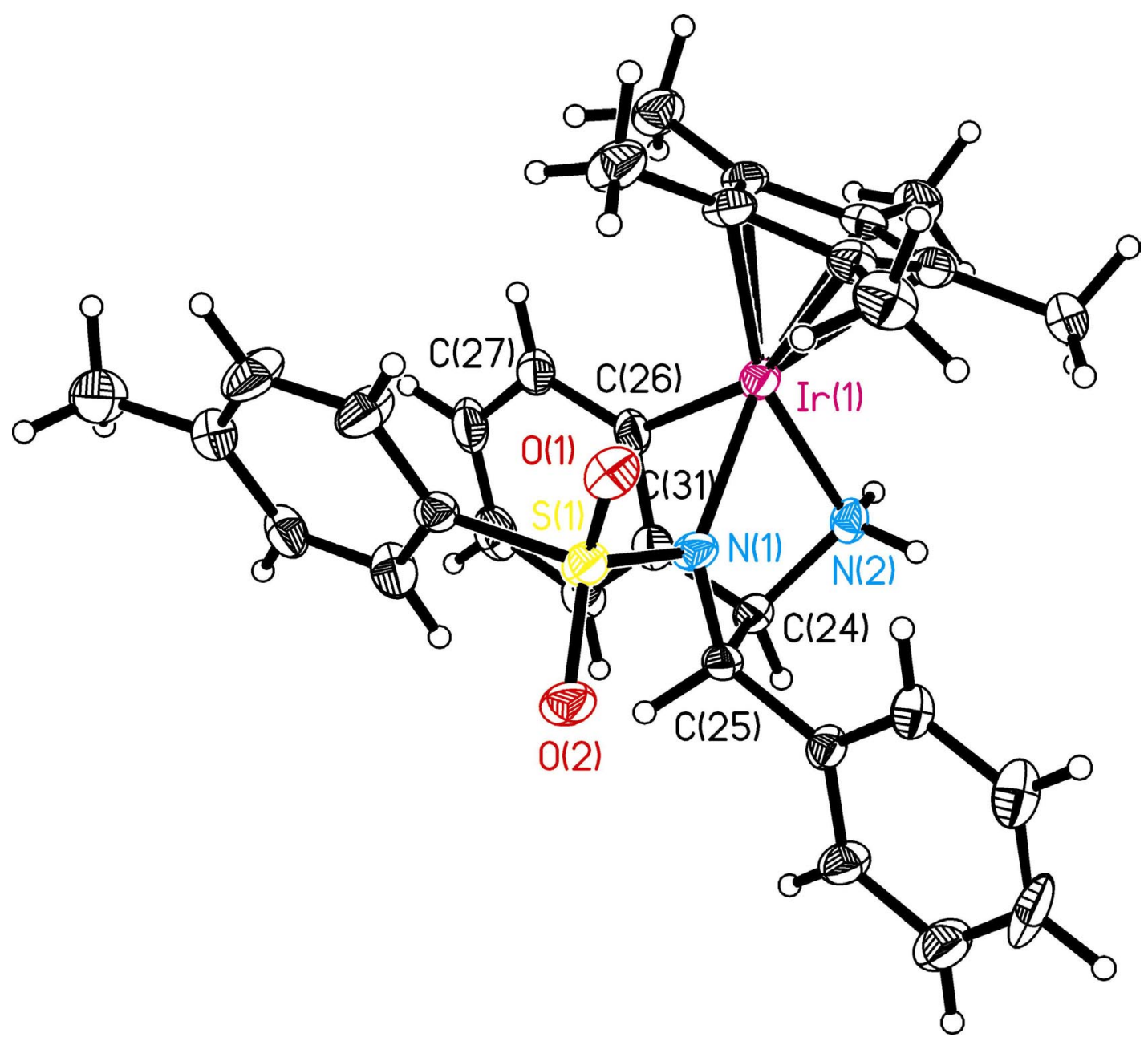

Figure S3: Molecular structure of 2. Thermal ellipsoids are shown at 50\% probability.

\section{5) References}

(1) Mashima, K.; Abe, T.; Tani, K. Chem. Lett. 1998, 1201-1202; Mashima, K.; Abe, T.; Tani, K. Chem. Lett. 1998, 1199-1200; Murata, K.; Ikariya, T.; Noyori, R. J. Org. Chem. 1999, 64, 2186-2187.

(2) Katayama, T.; Nitta, T. J. Chem. Eng. Data 1976, 21, 194-6.

(3) Lankamp, H.; Nauta, W. T.; MacLean, C. Tetrahedron Lett. 1968, 249-54.

(4) Kaljurand, I.; Kuett, A.; Soovaeli, L.; Rodima, T.; Maeemets, V.; Leito, I.; Koppel, I. A. J. Org. Chem. 2005, 70, 1019-1028.

(5) Schaefgen, J. R.; Newman, M. S.; Verhoek, F. H. J. Am. Chem. Soc. 1944, 66, 1847-9. 